\title{
Identification of HPV Integration and Genomic Patterns Delineating the Clinical Landscape of Cervical Cancer
}

\author{
Raid-Al Akeel*
}

\begin{abstract}
Cervical cancer is one of the most common cancers in women worldwide. During their life time the vast majority of women become infected with human papillomavirus (HPV), but interestingly only a small portion develop cervical cancer and in the remainder infection regresses to a normal healthy state. Beyond HPV status, associated molecular characterization of disease has to be established. However, initial work suggests the existence of several different molecular classes, based on the biological features of differentially expressed genes in each subtype. This suggests that additional risk factors play an important role in the outcome of infection. Host genomic factors play an important role in the outcome of such complex or multifactor diseases such as cervical cancer and are also known to regulate the rate of disease progression. The aim of this review was to compile advances in the field of host genomics of HPV positive and negative cervical cancer and their association with clinical response.
\end{abstract}

Keywords: Cervical cancer - HPV integration - genomic pattern

Asian Pac J Cancer Prev, 16 (18), 8041-8045

\section{Introduction}

Human papillomavirus (HPV) is one of the most widely publicized and researched pathogenic DNA viruses. For decades, HPV research has focused on transforming viral activities in cervical cancer (Allie et al., 2014). Cervical cancer is the most common cancers in women worldwide and Genomic instability is a hallmark of human cancers, including the $5 \%$ caused by human papillomavirus (HPV) (Keilo et al., 2013) . Cancer of the cervix is the second most common life-threatening cancer among women worldwide and both incidence and mortality rates are likely to be underestimated in developing countries. HPV high risk strains play at least the major if not an absolutely necessary role in the etiology (Behtash and Mehrdad, 2006). Persistent infection by ' $h i g h$ risk' genotypes of human papilloma virus (HPV) is necessary but not sufficient for the development of over $98 \%$ of cervical cancers (Ursula et al., 2006).

Clinical, epidemiological, and molecular data have clearly demonstrated that certain human papilloma viruses (HPVs) types, so-called high risk, are the etiological agents of cervical cancer (zur Hausen, 2002). The progress of cervical cancer is driven by infection with human papillomavirus (HPV) (Walboomers et al., 1999) through regulation of $\mathrm{p} 53$ and $\mathrm{Rb}$ tumor suppressor pathways by the viral oncoproteins E6 and E7 (Dyson et al., 1989; Band et al., 1991). Loss of p53 checkpoint function leads to genomic instability and, in addition to the initiating virusdriven events, cervical carcinomas accumulate numerous genomic changes, of which only some are driver mutations for tumor growth (Allen et al., 2000; Lando et al., 2009). These cancers are tough to treat and treatment can cause severe, long-term side effects. For patients who are not cured by surgery and/or chemo/radiotherapy, there are few effective treatment options. Molecular personalized treatments and predictive biomarkers are urgently needed in order to improve the management and minimize the toxicity, and to allow selection of patients who are likely to benefit from both nonselective and targeted treatments.

Though, there has been a alarming increase in the incidence rates of cervical cancers because of infection with high-risk human papillomavirus (HPV) (Chaturvedi et al., 2008; Chaturvedi et al., 2013). At present, treatment of an individual cancer is typically determined in a multidisciplinary setting, with the histological subtype, sub-site, staging information, patient fitness, baseline swallow and airway function guiding management decisions. About one third of patients present with earlystage disease and these patients are treated with either surgery or radiotherapy depending on the primary tumor site, with cure rates of 70-90\% (Argiris et al., 2008). However, the majority of patients present with locally advanced disease.

Fundamental treatment in this situation requires multimodality therapy with surgery, commonly followed by postoperative radiotherapy or chemoradiotherapy, or organ preserving primary radiotherapy, with or without chemotherapy (Pignon et al., 2009). These treatments are intensive and associated with severe acute toxicity, 
such as mucositis, dermatitis and dysphagia, and longterm sequelae, for example, sensorineural hearing loss, permanent xerostomia and altered swallowing function. In spite of recent advances in both surgical and radiotherapy delivery techniques, up to $50 \%$ of locally advanced tumors relapse usually within the first 2 years after treatment, with limited options for salvage surgery or re-irradiation (Argiris et al., 2008:Pignon et al., 2009). Many chemotherapy agents can be used for inoperable recurrences or metastatic disease, with response rates of only $10-35 \%$ and median survival of 6-12 months (Brockstein, 2011).

Beyond HPV status, associated molecular characterization of disease has to be established. However, initial work suggests the existence of several different molecular classes, based on the biological features of differentially expressed genes in each subtype (Walter et al., 2013). Genomic advances have shown new genes and pathways involved in the development and progression of cancer in general and cervical cancer in particular, creating prospects to explore novel personalized therapeutic targets. Cancer research has shifted to focus on biomarker discovery for diagnosis, prognosis and prediction of treatment response, alongside the development of personalized therapies, with the ultimate goal of individualizing therapy for each patient.

\section{HPV-Mediated Pathogenesis}

HPVs are small, non-enveloped, double-stranded DNA viruses. The genome encodes for early genes (E1-7) and late structural genes (L1,L2). E1 and E2 encode regulatory proteins and E5-7 encode oncoproteins. Over 100 human HPV genotypes have been isolated, and mucosal HPVs can be classified into high and low risk based on their potential to induce malignant transformation. HPVs infects the host via wounds or abrasions in the mucosa and infect basal epithelial cells, where it uses host cell DNA replication machinery for viral replication. The E6 protein interacts with E6-associated protein (E6-AP), resulting in a rapid degradation of tumor suppressor p53 pathway. This inhibits pro-apoptotic functions of $\mathrm{p} 53$ and bypasses p53-mediated checkpoints (Scheffner et al., 1990). The E7 protein competes with E2F transcription factor for binding to the $\mathrm{pRb}$ tumor suppressor, displacing E2F. E2F activates genes responsible for cell cycle progression through the G1 to $\mathrm{S}$ phase, including cyclin A, E and DNA polymerase, causing inactivation of checkpoints and regulatory pathways, and ultimately promoting cellular proliferation and transformation (Dyson et al., 1989). $\mathrm{pRb}$ is a negative regulator of the cyclin-dependent kinase inhibitor $\mathrm{p} 16$, and therefore inactivation of $\mathrm{pRb}$ results in p16 up regulation.

\section{TP53/RB Pathway}

Tumor suppressor protein p53 plays a key role in the regulation of genes involved in cell cycle and growth arrest, DNA repair or apoptosis, thereby maintaining genomic stability (Vogelstein et al., 2000). In response to DNA damage, p53 can arrest the cell cycle and activate repair or initiate apoptosis. p53 controls a significant spectrum of genes involved in various pathways (Brady et al., 2011); these include recently discovered biochemical pathways, such as the connection of IL-7Ra to telomere erosion (Kibe et al., 2012), the metabolism of the cell (Boren et al., 2012) and the silencing of repeats and noncoding RNA (Leonova et al., 2013).

Mutations in $\mathrm{p} 53$ and $\mathrm{pRb}$ pathways result in limitless replicative potential and immortalisation. TP53 mutations can occur throughout the entire gene but the majority are because of a missense mutation in the DNA-binding domain. These mutations can result in a number of consequences including inhibition of function, tumor suppressor loss or occasionally gain of function (Morton et al., 2010). In p53 wild-type tumors, p53 function may be inactivated by several mechanisms, such as HPV infection, overexpression or amplification of MDM2 and deletion of the p14 ${ }^{\mathrm{ARF}}$ gene (Vogelstein et al., 2000).

\section{Epidermal Growth Factor Receptor (EGFR) Pathway}

EGFR (ErbB1) is a member of the ErbB/HER2 family of transmembrane receptor tyrosine kinases. Other members include HER2 (ErbB2), ErbB3 and ErbB4 and they play a major role in cell proliferation, differentiation, survival and migration. EGFR is composed of an extracellular ligand-binding domain, a transmembrane segment and a cytoplasmic domain with tyrosine kinase activity. It is activated by a number of ligands including EGF, transforming growth factor- $\alpha$ and amphiregulin. Ligand binding results in a conformational change in EGFR and homo- or hetero-dimerisation with other ErbB family members, leading to autophosphorylation and receptor activation. This results in the activation of downstream signal transduction cascades including the Ras/Raf/mitogen-activated protein kinase (MAPK), phosphoinositide 3-kinase (PI3K)/AKT and Janus kinase (JAK)/signal transducer and activator of transcription 3 (STAT3) pathways (Hynes et al., 2005). The EGF-bound EGFR can also translocate to the nucleus to function as a transcription factor.

EGFR can be targeted either by inhibition of the extracellular ligand binding using monoclonal antibodies (mAbs), such as cetuximab, or by inhibition of the tyrosine kinase domain with a small molecule (TKIs), such as gefitinib, erlotinib and lapatinib. Cetuximab is a chimeric human-murine IgG1 mAb directed specifically against EGFR, resulting in inhibition of cell cycle progression, angiogenesis and metastasis, induction of apoptosis and synergy with radiotherapy and chemotherapy. Skin toxicity is a common side effect with cetuximab treatment and this clinical feature has been suggested as a biomarker for response to cetuximab, with response rates of $33 \%$ observed in patients with skin rash compared with $7 \%$ in those who do not develop skin toxicities (Burtness et al., 2005).

Panitumumab is a fully humanised $m A b$ against EGFR in use in colorectal cancer. In HNSCC, a phase III trial of panitumumab in combination with chemotherapy did not show an improvement in survival, although retrospective 
analysis showed that median overall survival in p16 (surrogate marker for HPV)-negative patients was longer in the panitumumab group than in the control group (Vermorken et al., 2013).

TKIs block the activation and phosphorylation of EGFR, and these drugs are given orally as they are well absorbed across the gastrointestinal tract. Gefitinib and erlotinib, currently used in lung cancer, inhibit only EGFR. Because of the potential resistance mechanisms, TKIs that have action against multiple ErbB family receptors are under investigation. Lapatinib has dual specificity for EGFR and HER2 and is in use in breast cancer. It has shown activity in p16-negative tumors in combination with chemoradiation (Harrington et al., 2013), and is currently being evaluated in the recurrent/metastatic setting in combination with capecitabine chemotherapy, and in a phase III adjuvant trial. Afatinib irreversibly blocks EGFR, HER2 and ErbB4 and is being investigated in the recurrent/metastatic, neoadjuvant and adjuvant settings of various cancers.

\section{NOTCH Pathway}

NOTCH1 signaling is involved in a number of biological functions, including regulation of self-renewal capacity, survival and promoting terminal differentiation. The NOTCH pathways consist of four receptors bound to the cell membrane, NOTCH 1-4, and two families of ligands, Delta-like (1, 3 and 4) and Jagged (1 and 2). Ligand binding leads to two cleavages of NOTCH1 by $\mathrm{TNF} \alpha$-converting enzyme (TACE) and $\gamma$-secretase, resulting in the release of NOTCH1 intracellular domain (NCID). NCID translocates to the nucleus to promote transcription of its target genes, including the HRT and HES families. NOTCH1 is regulated partly by ubiquitination and degradation that involves FBXW7 (Egloff and Grandis, 2012).

NOTCH1 signalling promotes terminal differentiation in keratinocytes and skin SCC, and this is negatively regulated by EGFR. Inhibition of EGFR blockade induces keratinocyte differentiation (Kolev et al., 2008). NOTCH1 has also been found to be inhibited in basal epithelial cells by the p53-related transcription factor p63 that becomes downregulated during terminal differentiation coinciding with NOTCH1 upregulation. Overexpression and amplification of TP63 have been observed in the majority of cancers (Dotto, 2009). However, since it is known that p63 encodes several isoforms with opposing functions, the precise role of p63 in NOTCH1 signalling and malignant transformation of oral epithelial cells remains to be elucidated.

NOTCH1 signalling has also been linked to suppression of HPV E6 and E7 protein expression in cervical carcinoma cell lines; expression of activated NOTCH1 causes growth inhibition of HPV-positive but not HPVnegative cervical carcinoma cell lines, and results in the downmodulation of HPV-driven transcription of the E6 and E7 viral genes ( Talora et al., 2002). Both NOTCH1 pathway inhibitors that inhibit $\gamma$-secretase and NOTCH1 pathway activators, via inhibition of histone deacetylase, are currently in clinical development.

\section{PI3K/AKT/mTOR Pathway}

PI3Ks are a family of enzymes that phosphorylate the $3 \mathrm{OH}$ position of phosphatidylinositols and have important roles in promoting cell growth, differentiation and survival. There are three classes of PI3Ks, each with its own substrate specificity, and class $1 \mathrm{~A}$ is most frequently associated with cancer. PI3Ks are activated by RTKs, such as EGFR, and the catalytic subunit phosphorylates phosphatidylinositol1, 4-bisphosphate $\left(\mathrm{PIP}_{2}\right)$ to form phosphatidylinositol1, 4, 5-triphosphate (PIP3). PIP3 recruits pleckstrin-homology domain-containing proteins including phosphoinositide-dependent protein-kinase 1 (PDK1) and AKT to the plasma membrane. Interaction of PIP3 with the PH (Pleckstrin Homology) domain of AKT results in a conformational change causing phosphorylation of AKT by PKD1 and mammalian target of rapamycin complex 2 (mTORC2). This activates AKT that then phosphorylates proteins involved in cell growth and survival. The tumor-suppressor phosphatase and tensin homology (PTEN) mediates the conversion of $\mathrm{PIP}_{3}$ to $\mathrm{PIP}_{2}$, counteracting the activation of AKT (Engelman et al ., 2009). mTOR is a protein kinase that acts downstream of PI3K and AKT and plays an important role in cell growth, survival and protein synthesis regulation.

Genetic aberrations of the PI3K pathway are common in cancers. One of the isoforms of the $110 \mathrm{kDa}$ catalytic subunit, $\mathrm{p} 110 \alpha$, is encoded by the PIK3CA gene. It has been found to be particularly common in HPV-positive cancer, and specific mutations, such as H1047R in exon 20, may predict higher response rates to treatment with PI3K pathway inhibitors (Qiu et al., 2006; Janku et al., 2013). In addition, PTEN mutations have been reported, and the mTOR pathway is frequently activated, independent from activation of EGFR or the presence of mutant p53, particularly in HPV-positive tumors (Molinolo et al., 2012).

PI3K pathway is an important therapeutic target for cancers and its therapeutic modulation has been assessed in a number of tumor types. The mTOR inhibitor everolimus is in clinical use in renal cell carcinoma, pancreatic neuroendocrine tumors, breast cancer and subependymal giant cell astrocytoma, and temsirolimus can be used in renal cell carcinoma. PI3K inhibitors are being investigated in phase II trials in conjunction with chemotherapy or cetuximab; AKT inhibitors are being tested in recurrent or metastatic nasopharyngeal cancer; and the mTOR inhibitors rapamycin, everolimus and temsirolimus are being assessed for HNSCC at the phase II stage in neoadjuvant and recurrent/metastatic settings.

\section{Ras/Raf/MEK/MAPK Pathway}

Ras is a guanosine nucleotide binding protein localised on the plasma membrane. There are three Ras genes: $H R A S, K R A S$ and NRAS. In the inactivated state, Ras is bound to guanosine diphosphate (GDP) and activation converts Ras to the guanosine triphosphate (GTP)-bound form; Ras-GTP binds to and activates Raf-1. The targets for phosphorylation of Raf-1 include the kinases MEK1 and MEK2 that in turn activate the MAP kinases ERK1 
and ERK2. These translocate to the nucleus and target genes involved in cell growth, proliferation and survival. Ras can also activate the PI3K signalling cascade (Bos, 1989).

Mutations in the Ras proto-oncogenes are implicated in $20-30 \%$ of all cancers . Activating HRAS mutations have been found in $4-5 \%$ of HNSCC cases 20, 27). KRAS mutations occur in $30-50 \%$ of colorectal cancers and are predictive of poor response to panitumumab and cetuximab (Bardelli and Siena, 2010). Sorafenib is a tyrosine kinase inhibitor that has multiple targets including Raf, VEGF (vascular endothelial growth factor receptor) and PDGFR (platelet-derived growth factor receptor) (Wilhelm et al., 2008). It is in use in renal cell carcinoma and hepatocellular carcinoma. Sorafenib in combination with chemotherapy has shown a response rate of $55 \%$ and median overall survival of 22.6 months in a phase II trial in HNSCC (Blumenschein et al., 2012). The MEK inhibitor trametinib has recently been approved for use in metastatic melanoma and is under investigation in combination with AKT inhibition in solid tumors including HNSCC.

\section{MET Pathway}

The proto-oncogene c-MET encodes mesenchymalepithelial transition factor (MET), an RTK activated by hepatocyte growth factor (HGF). Ligand binging activates signalling cascades including the RAS, PI3K, STAT3 and NOTCH pathways, resulting in cell morphogenesis, motility, growth and survival. MET and HGF have been found to be overexpressed in various cancers and increased MET copy numbers in 13\% of HNSCC tumor samples (Knowles et al., 2009, Seiwert et al., 2009). MET expression has been suggested to be a prognostic biomarker in HPV-negative cancers with overexpression correlating with reduced disease-free and overall survival (Lo et al., 2006; Zhao et al., 2011). It has also been implicated in resistance to radiation, cisplatin and cetuximab (De et al., 2011; Krumbach et al., 2011; Sun and Wang, 2011).

MET overexpression results in enhanced cell motility, angiogenesis and invasion/metastases, and therefore is an important potential therapeutic target. Foretinib is a multi-tyrosine kinase inhibitor that binds to the adenosine triphosphate (ATP) pocket of the receptor. It has been tested in a phase II study in recurrent/metastatic HNSCC but showed disease stabilization and only minor tumor shrinkage as a single agent (Seiwert et al., 2013). There are several RTK inhibitors and mAbs against MET and HGF in early phase clinical trials.

\section{Conclusions}

Cervical cancer is highly heterogeneous tumors, therefore difficult to treat and management is likely to change in the near future, moving from treatment as a single disease to tailoring the therapy based on both patient and tumor characteristics. Identification of specific genomic pattern, together with the more traditional techniques in diagnosis, staging and prognostication. It has the potential to provide the clinician with a comprehensive set of diagnostic, prognostic and predictive tools. The paucity of driver genomic alterations in HPV associated cervical cancer and frequent tumor suppressor loss represents a pharmacological challenge, but increased understanding of the molecular biology through the developments in next generation sequencing technology heralds a future of personalized medicine.

\section{Acknowledgements}

I'm thankful to the Department of Clinical Laboratory Sciences, College of Applied Medical Sciences, King Saud University, Riyadh, Saudi Arabia for supporting me in compiling the work

\section{References}

Allie K Adams, Trisha M Wise-Draper, Susanne I (2014). Wells human papillomavirus induced transformation in cervical and head and neck cancers. Cancers (Basel), 6; 1793-820.

Allen DG, White DJ, Hutchins AM, et al (2000). Progressive genetic aberrations detected by comparative genomic hybridization in squamous cell cervical cancer. Br J Cancer, 83, 1659-63.

Argiris A, Karamouzis MV, Raben D, Ferris RL (2008). Head and neck cancer. Lancet, 371, 1695-709.

Brady CA, Jiang D, Mello SS, et al (2011). Distinct p53 transcriptional programs dictate acute DNA-damage responses and tumor suppression. Cell, 145, 571-583.

Boren J, Brindle KM (2012). Apoptosis-induced mitochondrial dysfunction causes cytoplasmic lipid droplet formation. Cell Death Differ, 19, 1561-70.

Burtness B, Goldwasser MA, Flood W, Mattar B, Forastiere AA (2005). Eastern cooperative oncology g. phase iii randomized trial of cisplatin plus placebo compared with cisplatin plus cetuximab in metastatic/recurrent head and neck cancer: an eastern cooperative oncology group study. J Clin Oncol, 23, 8646-54.

Band V, De Caprio J, Delmolina L, Kulesa V, Sager R (1991). Loss of p53 protein in Human papillomavirus type 16 E6immortalised human mammary epithelial cells. J Virol, 65, 6671-6.

Behtash N, Mehrdad N (2006). Cervical cancer: screening and prevention. Asian Pac J Cancer Prev, 7, 683-6

Bos JL (1989). ras oncogenes in human cancer: a review. Cancer Res, 49, 4682-9.

Bardelli A, Siena S (2010). Molecular mechanisms of resistance to cetuximab and panitumumab in colorectal cancer. J Clin Oncol, 28, 1254-61.

Blumenschein GRJr, Glisson BS, Lu C, et al (2012). Final results of a phase II study of sorafenib in combination with carboplatin and paclitaxel in patients with metastatic or recurrent SCCHN. J Clin Oncol, 30, 5592.

Brockstein BE (2011). Management of recurrent head and neck cancer: recent progress and future directions. Drugs, 71, 1551-9.

Chaturvedi AK, Anderson WF, Lortet-Tieulent J, et al (2013). Worldwide trends in incidence rates for oral cavity and oropharyngeal cancers. J Clin Oncol, 31, 4550-9.

Chaturvedi AK, Engels EA, Anderson WF, Gillison ML (2008). Incidence trends for human papillamavirus-related and -unrelated oral squamous cell carcinomas in the United States. J Clin Oncol, 26, 612-9.

Dotto GP (2009). Crosstalk of notch with p53 and p63 in cancer growth control. Nat Rev Cancer, 9, 587-95. 
De Bacco F, Luraghi P, Medico E, et al (2011). Induction of MET by ionizing radiation and its role in radioresistance and invasive growth of cancer. J Natl Cancer Inst, 103, 645-61.

Dayson N, Howley PM, Munger K, Harlow E (1989). The human papilloma virus-16 E7 oncoprotein is able to bind to the retinoblastoma gene product. Science, 243, 934-937.

Egloff AM, Grandis JR (2012). Molecular pathways: contextdependent approaches to notch targeting as cancer therapy. Clin Cancer Res, 18, 5188-95.

Engelman JA (2009). Targeting PI3K signalling in cancer: opportunities, challenges and limitations. Nat Rev Cancer, 9, 550-62.

Hynes NE, Lane HA (2005). ERBB receptors and cancer: the complexity of targeted inhibitors. Nat Rev Cancer, 5, 341354.

Harrington K, Berrier A, Robinson M, et al (2013). Randomised phase II study of oral lapatinib combined with chemoradiotherapy in patients with advanced squamous cell carcinoma of the head and neck: rationale for future randomised trials in human papilloma virus-negative disease. Eur J Cancer, 49, 1609-18.

Janku F, Wheler JJ, Naing A, et al (2013). PIK3CA mutation $\mathrm{H} 1047 \mathrm{R}$ is associated with response to $\mathrm{PI} 3 \mathrm{~K} / \mathrm{AKT} / \mathrm{mTOR}$ signaling pathway inhibitors in early-phase clinical trials. Cancer Res, 73, 276-284.

Keiko Akagi, Jingfeng Li, Tatevik R Broutian, et al (2014). Genome-wide analysis of HPV integration in human cancers reveals recurrent, focal genomic instability. Genome Res, 24, 185-99.

Knowles LM, Stabile LP, Egloff AM, et al (2009). HGF and c-Met participate in paracrine tumorigenic pathways in head and neck squamous cell cancer. Clin Cancer Res, 15, 3740-50.

Kibe R, Zhang S, Guo D, et al (2012). IL-7Ralpha deficiency in p53null mice exacerbates thymocyte telomere erosion and lymphomagenesis. Cell Death Differ, 19, 1139-51.

Kolev V, Mandinova A, Guinea-Viniegra J, et al (2008). GFR signalling as a negative regulator of Notch1 gene transcription and function in proliferating keratinocytes and cancer. Nat Cell Biol, 10, 902-11

Krumbach R, Schuler J, Hofmann M, et al (2011). Primary resistance to cetuximab in a panel of patient-derived tumor xenograft models: activation of MET as one mechanism for drug resistance. Eur J Cancer, 47, 1231-43.

Leonova KI, Brodsky L, Lipchick B, et al (2013).p53 cooperates with DNA methylation and a suicidal interferon response to maintain epigenetic silencing of repeats and noncoding RNAs. Proc Natl Acad Sci USA, 110, 89-98.

Lo Muzio L, Farina A, Rubini C, et al (2006). Effect of c-Metexpression on survival in head and neck squamous cell carcinoma. Tumor Biol, 27, 115-121.

Lando M, Holden M, Bergersen LC, et al (2009). Gene dosage, expression, and ontology analysis identifies driver genes in the carcinogenesis and chemoradioresistance of cervical cancer. PLoS Genet, 5, 1000719.

Molinolo AA, Marsh C, El Dinali M, et al (2012). mTOR as a molecular target in HPV-associated oral and cervical squamous carcinomas. Clin Cancer Res, 18, 2558-68.

Morton JP, Timpson P, Karim SA, et al (2010). Mutant p53 drives metastasis and overcomes growth arrest/senescence in pancreatic cancer. Proc Natl Acad Sci USA, 107, 246-51

Pignon JP, le Maitre A, Maillard E, Bourhis J, Group M-NC (2009). Meta-analysis of chemotherapy in head and neck cancer (MACH-NC): an update on 93 randomised trials and 17, 346 patients. Radiother Oncol, 92, 4-14.

Qiu W, Schonleben F, Li X, et al (2006). PIK3CA mutations in head and neck squamous cell carcinoma. Clin Cancer Res,
12, 1441-6.

Seiwert TY, Jagadeeswaran R, Faoro L, et al (2009). The MET receptor tyrosine kinase is a potential novel therapeutic target for head and neck squamous cell carcinoma. Cancer Res, 69, 3021-31.

Sun S, Wang Z (2011). Head neck squamous cell carcinoma c-Met(+) cells display cancer stem cell properties and are responsible for cisplatin-resistance and metastasis. Int $J$ Cancer, 129, 2337-48.

Seiwert T, Sarantopoulos J, Kallender H, et al (2013). Phase II trial of single-agent foretinib (GSK1363089) in patients with recurrent or metastatic squamous cell carcinoma of the head and neck. Invest New Drugs, 31, 417-24.

Scheffner M, Werness BA, Huibregtse JM, Levine AJ, Howley PM (1990). The E6 oncoprotein encoded by human papillomavirus types 16 and 18 promotes the degradation of p53. Cell, 63, 1129-36.

Talora C, Sgroi DC, Crum CP, Dotto GP (2002). Specific downmodulation of Notch1 signaling in cervical cancer cells is required for sustained HPV-E6/E7 expression and late steps of malignant transformation. Genes Dev, 16, 2252-63.

Ursula Winters, Richard Roden, Henry Kitchener, Peter Stern (2006). Progress in the development of a cervical cancer vaccine. Ther Clin Risk Manag, 2, 259-69.

Vogelstein B, Lane D, Levine AJ (2000). Surfing the p53 network. Nature, 408, 307-310.

Vermorken JB, Stohlmacher-Williams J, Davidenko I, et al (2013). Cisplatin and fluorouracil with or without panitumumab in patients with recurrent or metastatic squamous-cell carcinoma of the head and neck (SPECTRUM): an openlabel phase 3 randomised trial. Lancet Oncol, 14, 697-710.

Walter V, Yin X, Wilkerson MD, et al (2013). Molecular subtypes in head and neck cancer exhibit distinct patterns of chromosomal gain and loss of canonicalcancer genes. PLoS One, 8, 56823. I

Wilhelm SM, Adnane L, Newell P, et al (2008). Preclinical overview of sorafenib, a multikinase inhibitor that targets both Raf and VEGF and PDGF receptor tyrosine kinase signaling. Mol Cancer Ther, 7, 3129-40.

Walboomers JM, Jacobs MV, Manos MM, et al (1999). Human papillomavirus is a necessary cause of invasive cervical cancer worldwide. J Pathol, 189, 12-19.

zur Hausen H (2002). Papillomaviruses and cancer: from basic studies to clinical application. Nat Rev Cancer, 2, 342-50

Zhao D, Wang SH, Feng Y, et al (2011). Intratumoral c-Met expression is associated with vascular endothelial growth factor $\mathrm{C}$ expression, lymphangiogenesis, and lymph node metastasis in oral squamous cell carcinoma: implications for use as a prognostic marker. Hum Pathol, 42, 1514-23. 